\title{
ON BROCKETT'S NECESSARY CONDITION FOR STABILIZABILITY AND THE TOPOLOGY OF LIAPUNOV FUNCTIONS ON $\mathbb{R}^{N *}$
}

\author{
CHRISTOPHER I. BYRNES ${ }^{\dagger}$
}

\begin{abstract}
In [2], Roger Brockett derived a necessary condition for the existence of a feedback control law asymptotically stabilizing an equilibrium for a given nonlinear control system. The intuitive appeal and the ease with which it can be applied have made this criterion one of the standard tools in the study of the feedback stabilizability of nonlinear control systems. Brockett's original proof used an impressive combination of Liapunov theory and algebraic topology, in part to cope with a lacuna in our understanding of the topology of the sublevel sets of Liapunov functions. In [33], F. W. Wilson, Jr. extended the converse theorems of Liapunov theory to compact attractors and proved some fundamental results about the topology of their domain of attraction and the level sets of their Liapunov functions. In particular, Wilson showed that the level sets $M_{c}=V^{-1}(c)$ are diffeomorphic to $S^{n-1}$ for $n \neq 4,5$ using the proof of the generalized Poincaré Conjecture of Smale. He observed that the excluded cases would from the validity of the Poincaré Conjecture in dimension 3 and 4 and showed that, for $n=5$, the assertion $\partial M_{c} \simeq S^{4}$ would imply the Poincaré Conjecture for 4-manifolds. Of course, the topological Poincaré Conjecture for $S^{4}$ was subsequently proved by Freedman in 1980 and with the remarkable recent solution by Perelman of the classical Poincaré Conjecture, Wilson's Theorem now holds for all $n$.

In this paper we describe the sublevel, and therefore as a corollary the level, sets of proper smooth functions $V: \mathbb{R}^{n} \rightarrow \mathbb{R}$ having a compact $\operatorname{set} \mathcal{C}(V)$ of critical points. Among the main results in this paper is the assertion that an arbitrary sublevel set $\mathcal{M}_{c}=V^{-1}[0, c]$ of such a function is homeomorphic to $\mathbb{D}^{n}$, the unit disk. For $n=2$, this assertion is a consequence of the Schönflies Theorem, a classical enhancement of the Jordan Curve Theorem. For arbitrary $n$ it follows from the generalized Schönflies Theorem of Mazur and Brown, from [33] and from the verification of the Poincaré Conjecture in all dimensions by Perelman, Freedman and Smale. We also describe the smooth structure of $\mathcal{M}_{c}$ and its boundary, generalizing the results of [33].

This result has several corollaries. In particular, using the Brouwer Fixed Point Theorem this gives a straightforward proof of Brockett's criterion and some of its enhancements to global attractors. These results in turn imply a new necessary condition for Input-to State Stability with respect to a compact set and an extension of Brockett's Theorem to the practical stabilizability of equilibria. Our main results can be further enhanced using the Poincaré-Hopf Theorem and, in this way, also lead to a streamlined version of Coron's proof [7] that Brockett's Theorem holds for continuous feedback laws, using a classical topological argument on the unit disc $\mathbb{D}^{n}$.
\end{abstract}

1. Introduction. Consider a control system

$$
\dot{x}=f(x, u) \quad x \in \mathbb{R}^{n}, u \in \mathbb{R}^{m}
$$

*Dedicated to my friend and teacher, Roger Ware Brockett, on the occasion of his seventieth birthday. Research supported in part by grants from the AFOSR.

$\dagger$ Department of Electrical and Systems Engineering, Washington University. Email:chrisbyrnes@wustl.edu 
where the vector field $f: \mathbb{R}^{n} \rightarrow \mathbb{R}^{n}$ is $\mathcal{C}^{1}$. Given a point $x_{0} \in \mathbb{R}^{n}$, a problem of great theoretical and practical interest is to find a $\mathcal{C}^{1}$ feedback control law

$$
u=u(x)
$$

rendering $x_{0} \in \mathbb{R}^{n}$ a locally asymptotically stable equilibrium for the closed-loop system

$$
\dot{x}=F_{u}(x):=f(x, u(x))
$$

Without loss of generality, we assume $x_{0}=0$. In [2], Roger Brockett proved a fundamental result concerning feedback stabilization.

Theorem 1.1. (Brockett) A necessary condition for the existence of a $\mathcal{C}^{1}$ feedback law (1.2) rendering $x_{0} \in \mathbb{R}^{n}$ locally asymptotically stability for the closed-loop system (1.3) is that

$$
f(x, u)=y, \text { for all }\|y\| \text { sufficiently small }
$$

be solvable for all $\|y\|$ sufficiently small.

Brockett's proof uses a combination of Liapunov theory and fixed point theory, an approach which has quite a few other corollaries as well. The basic idea is to prove that solvability of

$$
F(x)=y, \quad \text { for all }\|y\| \text { sufficiently small. }
$$

is a necessary condition for a $\mathcal{C}^{1}$ vector field

$$
\dot{x}=F(x), \quad F(0)=0,
$$

on $\mathbb{R}^{n}$ to have 0 as a locally asymptotically stable equilibrium. Indeed, if 0 as a locally asymptotically stable equilibrium for (1.6) then the domain of attraction, $\mathcal{D} \subset \mathbb{R}^{n}$, of 0 , is an open connected set on which $F$ generates a semiflow

$$
\Phi: \mathbb{R}^{+} \cup\{0\} \times \mathcal{D} \rightarrow \mathcal{D}, \quad \Phi_{t}(x):=\Phi(t, x) .
$$

Moreover, the classical converse $[17,18,33]$ to Liapunov's Theorem is the assertion that there exists a $\mathcal{C}^{\infty}$ Liapunov function

$$
V: \mathcal{D} \rightarrow \mathbb{R}
$$

for (1.6) that is proper, i.e., $V^{-1}[a, b]$ is compact for every closed interval $[a, b] \subset \mathbb{R}$. In this case, the level sets

$$
M_{c}=V^{-1}(c), \text { for } c>0,
$$

of $V$ are $\mathcal{C}^{\infty}$ compact manifolds. Similarly, the sublevel sets

$$
\mathcal{M}_{c}=V^{-1}(-\infty, c], \text { for } c>0
$$


are $\mathcal{C}^{\infty}$ compact manifolds with boundary, which are positively invariant under $F$ and therefore under $\Phi_{t}$, for all $t \geq 0$.

REMARK 1.1. We can rescale the vector field (1.6) by an integrating factor so that the rescaled trajectories are those of a flow $\bar{\Phi}: \mathbb{R}^{+} \cup\{0\} \times \mathcal{D} \rightarrow \mathcal{D}$ of a vector field $\bar{F}$ on $\mathcal{D}$. Since $\bar{F}$ is a complete vector field on the $n$-manifold $\mathcal{D}$ with a globally asymptotically stable equilibrium, $\mathcal{D}$ is diffeomorphic to $\mathbb{R}^{n}$ by a theorem of Milnor [20]. In particular, there is no difference between the topology of Liapunov functions for a locally versus a globally asymptotically stable equilibrium.

Remark 1.2. ([5]) Denote the interior of $\mathcal{M}_{c}$ by $\dot{\mathcal{M}}_{c}$. Since $\bar{\Phi}: \mathbb{R} \times \mathcal{M}_{c} \rightarrow \mathcal{M}_{c}$, by Milnor's Theorem we know that $\mathcal{M}_{c} \simeq \mathbb{R}^{n}$. Moreover, since $\mathcal{M}_{c}$ is a smooth $n$-manifold with boundary having a contractible interior, $\mathcal{M}_{c}$ is itself contractible [31, p. 297].

To say that (1.5) is solvable for a fixed $y$ is to say the vector field $\tilde{F}=F-y$ has an equilibrium. By continuity, the semiflow $\tilde{\Phi}$ of $\tilde{F}$ also leaves $\mathcal{M}_{c}$ positively invariant for $\|y\|$ sufficiently small and, by [35], the existence of an equilibrium for $\tilde{F}$ is equivalent to the existence of a fixed point for

$$
\tilde{\Phi}_{t}: \mathcal{M}_{c} \rightarrow \mathcal{M}_{c}
$$

for $t$ sufficiently small.

Recall $[9$, p. 2] that a topological space $X$ is said to be a fixed point space if every continuous map $f: X \rightarrow X$ has a fixed point. In this language, Theorem 1.1 will follow once we show that a sublevel set of a Liapunov function for an asymptotically stable equilibrium is always a fixed point space. Brockett's original proof [2] uses the fact that $\mathcal{M}_{c}$ is contractible. Compact contractible manifolds with boundary are fixed point spaces by the Lefschetz Fixed Point Theorem,[31, p. 196] - a far-reaching generalization of Brouwer's Fixed Point Theorem for closed balls in $\mathbb{R}^{n}$. In [29], Sontag gives a nice proof that every such $\mathcal{M}_{c}$ is a retract of a closed ball. As a retract of fixed point space, $\mathcal{M}_{c}$ is also a fixed point space [9, p. 3].

One of the corollaries of the main result in our paper is that each $\mathcal{M}_{c}$ is, in fact, homeomorphic to the $n$-disc, $\mathbb{D}^{n}$. In the equilibrium case, if a Liapunov function $V$ had the origin as a nondegenerate minimum, then the Morse Lemma would imply that, after a smooth local change of coordinates,

$$
V\left(x_{1}, \ldots, x_{n}\right)=x_{1}^{2}+\cdots+x_{n}^{2} .
$$

It follows that for $0<c \leq c_{1}$ sufficiently small, $\mathcal{M}_{c} \simeq \mathbb{D}^{n}$. Since $\nabla V(x) \neq 0$ for $x \neq 0$, integrating the vector field $\nabla V$ provides a diffeomorphism $\mathcal{M}_{c_{1}} \simeq \mathcal{M}_{c_{2}}$ for $c_{1}<c_{2}$, literally growing $\mathbb{R}^{n}$ along the integral curves of $\nabla V$. Of course, we are mainly interested in the case of critically stable equilibria and, while classical Morse Theory does not apply directly, it does suggest that $\mathcal{M}_{c}$ should be an $n$-cell, for $c$ sufficiently large. In fact, it is. 
Theorem 1.2. Suppose $V: \mathbb{R}^{n} \rightarrow \mathbb{R}$ is a smooth, proper function and the subset, $\mathcal{C}(V) \subset \mathbb{R}^{n}$, of critical points of $V$ is compact. For any $c>\max _{x \in \mathcal{C}(V)}\|x\|, \mathcal{M}_{c}$ is homeomorphic to $\mathbb{D}^{n}$. In particular, $\mathcal{M}_{c}$ is a fixed point space.

REMARK 1.3. If $F$ is locally Lipschitz and 0 is locally asymptotically stable, then Zabczyk [36] has given a proof of Theorem 1.1 using an index formula due to Krasnosel'skii [16]. Starting with Zabczyk's index criterion and using topological degree theory, Coron [7] has generalized Brockett's Theorem to the case of continuous feedback control laws (see Section 7).

EXAMPLE 1.1. [5] Choosing principal axes (i.e., diagonalizing the inertia matrix $J)$, the equations of motion for a rigid spacecraft with $m$ momentum exchange devices can be expressed in local coordinates about a reference frame $R=\left[r_{1}, r_{2}, r_{3}\right]$ using Euler angles $\phi, \theta, \psi$ representing rotations about the $r_{1}, r_{2}, r_{3}$ axes, respectively, with corresponding angular momentum variables $\omega_{1}, \omega_{2}, \omega_{3}$. This leads to the control system

$$
\begin{aligned}
& \left(\begin{array}{c}
\dot{\omega}_{1} \\
\dot{\omega}_{2} \\
\dot{\omega}_{3}
\end{array}\right)=\left(\begin{array}{c}
a_{1} \omega_{2} \omega_{3} \\
a_{2} \omega_{1} \omega_{3} \\
a_{3} \omega_{1} \omega_{2}
\end{array}\right)+\sum_{i=1}^{m} b_{i} u_{i}, \quad a_{i} \in \mathbb{R} \text { and } b_{i} \in \mathbb{R}^{3} \\
& \left(\begin{array}{c}
\dot{\phi} \\
\dot{\theta} \\
\dot{\psi}
\end{array}\right)=\left(\begin{array}{ccc}
\cos (\theta) & 0 & \sin (\theta) \\
\sin (\theta) \tan (\phi) & 1 & -\cos (\theta) \tan (\phi) \\
-\sin (\theta) \sec (\phi) & 0 & \cos (\theta) \sec (\phi)
\end{array}\right)\left(\begin{array}{l}
\omega_{1} \\
\omega_{2} \\
\omega_{3}
\end{array}\right)
\end{aligned}
$$

for which we set $\mathcal{B}=\operatorname{span}_{\mathbb{R}}\left\{b_{1}, \ldots, b_{m}\right\}$ and $\widetilde{m}=\operatorname{dim}_{\mathbb{R}} \mathcal{B}$. It can be shown explicitly [12] that when $\widetilde{m} \geq 2$ the system (1.11) - (1.12) satisfies the accessibility rank condition for a generic plane $\mathcal{B}$ and, in this case, is small-time locally controllable [7]. On the other hand, a straightforward calculation shows that (1.4) is solvable for all $y$ having the form

$$
y=\left(\begin{array}{c}
y_{1} \\
y_{2} \\
y_{3} \\
0 \\
0 \\
0
\end{array}\right) \text { where }\left|y_{i}\right| \text { sufficiently small }
$$

if and only if

$$
\widetilde{m}=3
$$

Therefore, there exists a control law (1.2) locally asymptotically stabilizing the origin if and only if (1.13) holds, or equivalently [5], if and only if the system (1.11)-(1.12) is 
linearizable by state feedback. In particular, the generic underactuated $(\widetilde{m}=2)$ rigid body model is locally controllable but never continuously asymptotically stabilizable.

In Section 2, we recall some basic facts about compact attractors on $\mathbb{R}^{n}$ and present an example illustrating what Theorem 3.1 asserts and does not assert. We use Theorem 3.1 to prove Brockett's criterion using Brouwer's Fixed Point Theorem and review some examples from the literature. In Section 4, we prove an enhancement of Theorem 3.1 asserting that $\mathcal{M}_{c}$ is in fact diffeomorphic to $\mathbb{D}^{n}$, except perhaps when $n=4$. We also present a corollary recording the quantitative implications of the Lefschetz Fixed Point Formula and the fact $\mathcal{M}_{c} \simeq \mathbb{D}^{n}$. As is well-known, the Lefschetz Fixed Point Formula implies the Poincaré - Hopf Index Theorem and in Section 5 we delineate the implications of index theory for feedback stabilization, giving a new proof of Krasnosel'skii's index formula (Corollary 4.2), a necessary condition due to Zabczyk [36] and a review of some related results. We conclude with an extension of Brockett's necessary condition to the problem of practical stabilizability (Corollary $6.1)$.

It is a pleasure to thank Andrew Berman, Roger Brockett and Moe Hirsch for helpful remarks, and the referee and editors for several constructive suggestions.

2. Compact attractors in $\mathbb{R}^{n}$. Consider the vector field $F$ defined via (1.6) generating the semiflow (1.7). Following [10], for a choice of metric $d$ on $\mathbb{R}^{n}$, we shall say that a closed subset $A \subset \mathbb{R}^{n}$ attracts a closed subset $B \subset \mathbb{R}^{n}$ provided the distance between the sets $\Phi_{t}(B)$ and $A$, defined as

$$
\operatorname{dist}\left(\Phi_{\mathrm{t}}(\mathrm{B}), \mathrm{A}\right):=\sup _{\mathrm{y} \in \mathrm{B}} \inf _{\mathrm{x} \in \mathrm{A}} \mathrm{d}\left(\Phi_{\mathrm{t}}(\mathrm{y}), \mathrm{x}\right),
$$

tends to 0 as $t \rightarrow+\infty$.

Definition 2.1. [10] A compact invariant subset $J$ is said to

1. be stable provided for every neighborhood $V$ of $J$, there exists a neighborhood $V^{\prime}$ of $J$, satisfying $\Phi_{t}\left(V^{\prime}\right) \subset V$, for all $t \geq 0$;

2. attract points locally if there exists a neighborhood $W$ of $J$ such that $J$ attracts each point in $W$;

3. be asymptotically stable if $J$ is stable and attracts points locally;

REMARK 2.1. Since $J$ is compact, the notion of attracting a point or attracting a compact set is independent of the choice of metric, as it should be. Moreover, since $J$ is compact, condition (3) is equivalent to the existence of a positively invariant neighborhood $J \subset K$ for which $J$ attracts $K$ [10, Lemma 3.3.1].

Definition 2.2. A stable compact invariant set $J$ is globally asymptotically stable provided it attracts every compact subset of $\mathbb{R}^{n}$. In this case, we say that $J$ is a compact attractor for $F \in \operatorname{Vect}\left(\mathbb{R}^{n}\right)$.

For any $B \subset \mathbb{R}^{n}$, the $\omega$-limit set of $B$, as defined in [10], is

$$
\omega(B)=\left\{x \in B \mid \text { for } \mathrm{x}_{\mathrm{j}} \in \mathrm{B} \text { and } \mathrm{t}_{\mathrm{j}} \rightarrow+\infty, \text { with } \mathrm{j} \rightarrow+\infty, \Phi\left(\mathrm{t}_{\mathrm{n}}, \mathrm{x}_{\mathrm{n}}\right) \rightarrow \mathrm{x}\right\} .
$$


For $B=\{x\}$, this coincides with the omega limit set $\omega(x)$ introduced by Birkhoff in 1927. Adapting Theorem 3.4.2 of [10] to our setting yields the following fundamental result.

TheOREM 2.1. [10] Suppose $M \subset \mathbb{R}^{n}$ is a compact submanifold with boundary that is positively invariant for $F$. Then,

1. $\omega(M)$ is the maximal non-empty, compact invariant set for (1.7)

2. $\omega(M)$ is connected;

3. $\omega(M)$ is stable;

4. $\omega(M)$ attracts every compact subset of $M$.

Since $\omega(M)$ is compact and invariant, for each $x_{0} \in \omega(M)$, there exists a $K\left(x_{0}\right)>$ 0 such that

$$
\left\|\Phi\left(t, x_{0}\right)\right\| \leq K\left(x_{0}\right), \text { for all }-\infty<t<\infty
$$

Definition 2.3. A trajectory of (1.6) with initial condition $x_{0}$ is Lagrange stable if, and only if, (2.3) is satisfed for some $K\left(x_{0}\right)>0$.

Corollary 2.1. Suppose $M \subset \mathbb{R}^{n}$ is a compact, connected submanifold with boundary that is positively invariant for $F$. Then, $\omega(M)$ is a compact, connected Liapunov stable attractor for $F$ on $M$. Moreover,

$$
\omega(M)=\{\text { Lagrange stable trajectories in } M\}
$$

Definition 2.4. [[33]] Suppose $F$ leaves a domain $D$ invariant and that $J \subset D$ is compact. A Liapunov function $V$ for $F$ on the pair $(D, J)$ is a $\mathcal{C}^{\infty}$ function $V: D \rightarrow \mathbb{R}$ that satisfies

1. $\left.V\right|_{J}=0$ and $V(x)>0$ for $x \notin J$,

2. $\dot{V}<0$ on $D-J$, and

3. $V$ tends to a constant value (possibly $\infty$ ) on $\partial D$.

TheOREm 2.2 ([33]-[34]). A necessary and sufficient condition for a compact subset $J \subset \mathbb{R}^{n}$ to be globally asymptotically stable in an open invariant domain $D \subset \mathbb{R}^{n}$ is that there exist a Liapunov function $V$ for $f$ on the pair $(D, J)$.

Example 2.1. Consider the simple planar system

$$
\begin{aligned}
& \dot{r}=r(2-r) \\
& \dot{\theta}=1
\end{aligned}
$$

which has an unstable equilibrium at the origin and a locally asymptotically stable limit cycle $\gamma$ evolving on $r=2$. The function

$$
V(r, \theta)=(2-r)^{2}
$$

is a global Liapunov function for $\gamma$ on $M=\mathbb{R}^{2}-\{(0,0)\}$ and has as a sublevel set an annulus 


$$
\mathbb{A}=V^{-1}[0,1]
$$

Of course, $\gamma$ is not globally asymptotically on $\mathbb{R}^{2}$, since the equilibrium at the origin does not tend to $\gamma$ as $t \rightarrow \infty$

On the other hand, the compact invariant subset

$$
\mathcal{A}=\{(r, \theta): r \leq 2\}
$$

is a global compact attractor for $(2.5)$ on $\mathbb{R}^{2}$ and, indeed, consists of all Lagrange stable orbits. Moreover, suppose

$$
\phi: \mathbb{R} \rightarrow \mathbb{R}
$$

is any monotone nondecreasing function satisfying $\phi(x)=0$ for $x \leq 2$ and $\phi(x)=1$ for $x \geq 3$. A straightforward calculation shows that the function

$$
W(r, \theta)=\phi(r) V(r, \theta)
$$

is a Liapunov function for the pair $\left(\mathbb{R}^{2}, \mathcal{A}\right)$ and that

$$
W^{-1}[0, c] \simeq \mathbb{D}^{2}
$$

for any $c>0$. The sublevel sets of $W$ are 2 discs, in harmony with Theorem 3.1.

REMARK 2.2. As in Remark 1.2, the interior of any positively invariant neighborhood of a locally asymptotically stable equilibrium in $\mathbb{R}^{n}$ is diffeomorphic to $\mathbb{R}^{n}$. As this simple example shows, this remarkable fact does not hold in general for the topology of positively invariant neighborhood of a locally asymptotically stable attractor. This is key to understanding Theorem 3.1, which is an assertion about global attractors (see Remark 1.1).

3. The Topology of Liapunov Functions on $\mathbb{R}^{n}$. Our main theorem, Theorem 4.1, holds for sublevel sets of proper functions $V: \mathbb{R}^{n} \rightarrow \mathbb{R}$ having a compact set of critical points and has the following corollary for systems having compact global attractors. In this statement, the observations about $\mathcal{M}_{c}$ are new but the heart and soul of the corollary are due to fundamental work of Wilson [33] and to the solution of the Poincaré Conjecture in all dimensions ([26],[8],[24],[23]).

Theorem 3.1. Suppose that $\mathcal{A}$ is a stable compact global attractor for (1.6) and that $V$ is a Liapunov function for $\mathcal{A}$. For any $c>0, \mathcal{M}_{c}$ is homeomorphic to $\mathbb{D}^{n}$ and the smooth hypersurface $M_{c}$ is diffeomorphic to $S^{n-1}$.

Proof. For $n=2, M_{c} \simeq S^{1}$ and Theorem 3.1 follows from the Schönflies Theorem, which asserts that a smooth closed curve separates $\mathbb{R}^{2}$ into two connected components, with the interior being homeomorphic to $\mathbb{D}^{2}$. 
For $n=3, M_{c}$ is a closed, orientable surface and is therefore classified by its Euler characteristic [11]. In particular, using Krasnosel'skii's calculation [16] of the index of an asymptotically stable equilibrium and the Poincaré - Hopf Index Theorem, one sees that

$$
-1=\operatorname{Ind}_{0}(F)=\chi\left(\mathcal{M}_{c}\right)-\chi\left(M_{c}\right) .
$$

Since $\mathcal{M}_{c}$ is contractible, $\chi\left(\mathcal{M}_{c}\right)=1$ so that $\chi\left(M_{c}\right)=2$ and therefore $M_{c} \simeq S^{2}$. In the non-equilibrium case, we argue as follows. Since $\mathcal{M}_{c}$ is contractible, its boundary $M_{c}$ has the integral homology of $S^{2}$, by the Lefschetz Duality Theorem. Therefore, $\chi\left(M_{c}\right)=2$ and $M_{c} \simeq S^{2}$.

Remark 3.1. The Schönflies Theorem implies the Jordan Curve Theorem in $\mathbb{R}^{2}$, which has itself been generalized as the Jordan-Brouwer Separation Theorem for $\mathbb{R}^{n}$. It is worth noting, however, that there are examples [1] of "wildly embedded" spheres in $\mathbb{R}^{3}$ which do separate $\mathbb{R}^{3}$ into an inside and an outside, but for which the interior is not homeomorphic to $\mathbb{D}^{3}$.

However, since $M_{c}$ is a smooth submanifold, the tubular neighborhood theorem implies that $M_{c}$ is embedded in a bicollared manner and it follows from the generalized Schönflies Theorem of B. Mazur [19] and M. Brown [3] that $\mathcal{M}_{c}$ is homeomorphic to $\mathbb{D}^{3}$.

In general, the boundary of a compact contractible $n$-manifold is a homology $(n-1)$-sphere. For $n \geq 6$, it is known [25] that the mapping that assigns to any compact $n$-manifold $\mathcal{M}$ with boundary its boundary $\partial \mathcal{M}$ is a bijection from the set of compact contractible topological $n$-manifolds to the set of $(n-1)$-dimensional topological homology spheres. Therefore, for $n \geq 6$, Theorem 3.1 implies and is implied by the assertion that $M_{c}$ is homeomorphic to $S^{n-1}$. In fact, this latter result was proved by F. W. Wilson in [33] using Smale's proof [26] of the generalized Poincaré Conjecture via the $h$-Cobordism Theorem (see also [22]).

The starting point in [33] is the neat observation that if 0 is a globally asymptotically stable equilibrium for a complete vector field (1.6) and $V$ is a proper Liapunov function, then the flow $\Phi: \mathbb{R} \times \mathbb{R}^{n}-\{0\} \rightarrow \mathbb{R}^{n}-\{0\}$ provides a diffeomorphism

$$
M_{c} \times \mathbb{R} \simeq \mathbb{R}^{n}-\{0\} \simeq S^{n-1} \times \mathbb{R}^{+},
$$

so that

$$
\pi_{i}\left(M_{c}\right) \simeq \pi_{i}\left(S^{n-1}\right) \text {, for all } i \geq 1 .
$$

Therefore, $M_{c}$ is a homotopy sphere.

If (1.6) has a non-equilibrium attractor $\mathcal{A}$, then Wilson reverses time and smooths the vector field at $\infty$ to obtain a smooth vector field on $S^{n}-\mathcal{A}$ with a globally asymptotically stable equilibrium and a Liapunov function with $M_{c}$ as a level set. By Milnor's Theorem, $S^{n}-\mathcal{A} \simeq \mathbb{R}^{n}$ and therefore $M_{c}$ is a homotopy sphere. 
We record the results we need from [33] as a single theorem.

TheOREm 3.2. (Wilson) Suppose that $\mathcal{A}$ is a stable compact global attractor for (1.6) and that $V$ is a Liapunov function for $\mathcal{A}$. For any $c>0, M_{c}$ is a homotopy $(n-1)$-sphere. In particular, the compact hypersurface $M_{c} \subset \mathbb{R}^{n}$ is diffeomorphic to $S^{n-1}$, except perhaps when $n=4,5$.

Wilson observed that the excluded cases would from the validity of the Poincaré Conjecture in dimension 3 and 4 and, in fact, showed that for $n=5$ the assertion $\partial M_{c} \simeq S^{4}$ would imply the Poincaré Conjecture for 4-manifolds, a result which was unknown at the time.

The Poincaré Conjecture for $S^{4}$ was proved by Freedman [8] in 1980 as a corollary of his classification of simply-connected closed topological 4-manifolds. With the remarkable recent solution by Perelman [24],[23] of the classical Poincaré Conjecture in dimension 3, we now know that $M_{c}$ is diffeomorphic to $S^{n-1}$ for all $n \geq 1$.

Matters being so, the generalized Schönflies Theorem of B. Mazur [19] and M. Brown [3] implies that $\mathcal{M}_{c}$ is homeomorphic to $\mathbb{D}^{n}$, for the remaining cases, $n=4,5$.

In the light of Remark 1.1, Theorem 3.1 has the following corollary.

Corollary 3.1. Suppose that $x_{0}$ is a locally asymptotically stable equilibrium for (1.6) and that $V$ is Liapunov function for $x_{0}$ and (1.6) defined on an open neighborhood $x_{0} \in U$. For any $c>0$ small enough so that the sublevel set $\mathcal{M}_{c} \subset U$ is compact, $\mathcal{M}_{c}$ is homeomorphic to $\mathbb{D}^{n}$.

4. The Main Theorem and Some Corollaries. In this section, we shall prove the following result which, together with Theorem 2.2, implies Theorem 3.1.

Theorem 4.1. Suppose $V: \mathbb{R}^{n} \rightarrow \mathbb{R}$ is a smooth, proper function and the subset, $\mathcal{C}(V) \subset \mathbb{R}^{n}$, of critical points of $V$ is compact. For any $c>\max _{x \in \mathcal{C}(V)}\|x\|$ :

1. The vector field $\nabla V(x)$ points in every direction, as $x \in \mathcal{M}_{c}$ varies, and

2. $\mathcal{M}_{c}$ is homeomorphic to $\mathbb{D}^{n}$.

In fact, $\mathcal{M}_{c}$ is diffeomorphic to $\mathbb{D}^{n}$, except perhaps when $n=4$. Moreover, for all $n \geq 1, M_{c}$ is diffeomorphic to $S^{n-1}$.

Proof. The dynamical system

$$
\dot{x}=-\nabla V(x), \quad x \in \mathbb{R}^{n}
$$

defines a dissipative system on $\mathbb{R}^{n}$, in the sense of Hale [10], and has $M_{c}$ as a compact positively invariant absorbing set. In particular [10], there exists a compact Liapunov stable global attractor $\mathcal{A} \subset \mathcal{M}_{c}$ for (4.1). We note that $\mathbb{R}^{n}-\mathcal{A}$ is an invariant set of both (4.1) and the dynamical system

$$
\dot{x}=\nabla V(x) .
$$


Since $\mathbb{R}^{n} \subset S^{n}=\mathbb{R}^{n} \dot{\cup}\{\infty\}$, the integral curves of (4.2) lie in $M^{+}=S^{n}-\mathcal{A}$ and tend to $\infty \in S^{n}$ as $t \rightarrow \infty$. In particular, $\infty$ is a globally asymptotically stable equilibrium for a new vector field $X^{+}$on $M^{+}$obtained by smoothing the vector field $\nabla V$ to 0 as $x \rightarrow \infty$, in a small neighborhood of $\infty$, without changing the trajectories of (4.2) as oriented 1-manifolds, as in [33, p. 325].

By Remark 1.1, we know that $M^{+}$is diffeomorphic to $\mathbb{R}^{n}$. Matters being so, we are able to apply some of the fundamental results proved by F. W. Wilson in [33]. Suppose $W$ is a proper Liapunov function for $\left(M^{+},\{\infty\}\right)$ and that $\mathcal{N}_{\kappa}, \kappa>0$, denotes a sublevel set of $W$ and $N_{\kappa}=W^{-1}(\kappa)=\partial \mathcal{N}_{\kappa}$. By Theorem 3.2, $N_{\kappa}$ is a homotopy sphere.

The function $L: M^{+} \rightarrow \mathbb{R}$ defined via $L(x)=\mathrm{e}^{-V(x)}$ is continuous and is smooth on $M^{+}-\{\infty\}$. Since the non-equilibrium trajectories of $X^{+}$are orientation-preserving reparameterizations of the trajectories of (4.2), $\dot{L}<0$ on $M^{+}-\{\infty\}$. In fact, $X^{+}=$ $\nabla V$ on a tubular neighborhood of the compact hypersurface $M_{c}=L^{-1}\left(\mathrm{e}^{-c}\right)$. In particular, each trajectory of $X^{+}$intersects $M_{c}$ exactly once, transversely. Similarly, each trajectory of $X^{+}$intersects $N_{\kappa}$ exactly once, transversely. If $\kappa>0$ is chosen sufficiently small, $M_{c} \cap N_{\kappa}=\emptyset$ and for each $x \in M_{c}$ there exists a smooth, everywhere positive function $\tau(x)$ defined by the condition $\bar{\Phi}_{\tau(x)}(x) \in N_{\kappa}$. Therefore, the map $T: M_{c} \rightarrow N_{\kappa}$ defined by $T(x)=\bar{\Phi}_{\tau(x)}(x)$ is a diffeomorphism. This proves the following result:

Lemma 4.1. $M_{c}$ is a homotopy sphere.

REMARK 4.1. The proof of Lemma 4.1 is a technical modification of the proof of Theorem 3.2 in [33] that is needed to accommodate the fact that $V$ is not a Liapunov function for the attractor $\mathcal{A} \subset \mathcal{M}_{c}$ for the gradient dynamical system (4.1). Indeed, elementary examples show that $\mathcal{A}$ will in general contain Lagrange stable orbits, connecting the critical points of $V$, on which $V$ will not be constant. Indeed, the starting point in [33] is the existence of a stable compact global attractor $\mathcal{A}$ for (1.6) on $\mathbb{R}^{n}$ to study the topology of a proper Liapunov function $V$. In contrast, we start with a proper function $V$ with a compact set of critical points and use [10] to conclude the existence of a compact, Liapunov attractor, $\mathcal{A}$, for the gradient system (4.1) to study the topology of $V$. In fact, Hale [10] shows that $\mathcal{A}$ coincides with the set of Lagrange stable orbits.

We can now apply Wilson's Theorem 3.2 to conclude:

Lemma 4.2. Suppose $V: \mathbb{R}^{n} \rightarrow \mathbb{R}$ is a smooth, proper function and the subset, $\mathcal{C}(V) \subset \mathbb{R}^{n}$, of critical points of $V$ is compact. For any $c>\max _{x \in \mathcal{C}(V)}\|x\|, \mathcal{M}_{c}$, the level set $M_{c} \subset \mathbb{R}^{n}$ is a smoothly embedded $S^{n-1}$.

We now turn to the topology of $\mathcal{M}_{c}$.

Proposition 4.1. Suppose $V: \mathbb{R}^{n} \rightarrow \mathbb{R}$ is a smooth, proper function and the subset, $\mathcal{C}(V) \subset \mathbb{R}^{n}$, of critical points of $V$ is compact. For any $c>\max _{x \in \mathcal{C}(V)}\|x\|$, the sublevel set $\mathcal{M}_{c} \subset \mathbb{R}^{n}$ is a smoothly embedded $\mathbb{D}^{n}$, except perhaps when $n=4$. 
Proof. For $n \leq 3$, two $n$-manifolds are diffeomorphic if, and only if, they are homeomorphic. Therefore, Proposition 4.1 follows from Theorem 3.1 for $n \leq 3$. For $n \geq 5$, since $M_{c} \subset \mathbb{R}^{n}$ is a smoothly embedded $S^{n-1}$, Proposition 4.1 follows from the Differentiable Schönflies Theorem [22, Theorem D, p. 112].

Remark 4.2. The Differentiable Schönflies Theorem was proven by Smale [27, Corollary 1.3] for the cases $n \neq 4,7$. Suppose $n=7$. By Remark 1.2, $\mathcal{M}_{c}$ is homeomorphic to $\mathbb{R}^{7}$ and $\mathcal{M}_{c}$ is contractible. Since $\partial \mathcal{M}_{C}=M_{c} \simeq S^{6}$ is simply connected, the restriction $n=7$ can be removed by applying the subsequent results of Smale [28, Theorem 5.1], see also Milnor [22, p.108].

Proposition 4.2. If $n=4, \mathcal{M}_{c}$ is homeomorphic to $\mathcal{D}^{4}$.

Proof. $M_{c}$ is a smoothly embedded $S^{3}$, separating $\mathbb{R}^{4}$ into an inside, $\mathcal{M}_{c}$, and an outside. By the tubular neighborhood theorem, there exists a neighborhood $U$ of $M_{c}$ and a diffeomorphism $\Psi: U \rightarrow S^{3} \times[-1,1]$, with $\Psi\left(M_{c}\right)=S^{3} \times\{0\}$. In particular, $M_{c}$ is a "bicollared" embedded $S^{3}$ and therefore, by the generalized Schönflies Theorem of B. Mazur [19] and M. Brown [3], the closure of the interior of $M_{c}$, i.e. $\mathcal{M}_{c}$, is homeomorphic to $\mathbb{D}^{4}$.

Finally, choose any vector $v \in \mathbb{R}^{n}$ and consider the vector field $F_{\epsilon}(x)=-\nabla V(x)+$ $\epsilon v$. As before, for $\epsilon>0$ and sufficiently small, $F_{\epsilon}$ points inward on $M_{c}$ and therefore has an equilibrium $x_{\epsilon} \in \mathcal{M}_{c} \simeq \mathbb{D}^{n}$. Therefore, $\nabla V\left(x_{\epsilon}\right)$ points in the direction of $v$.

REMARK 4.3. The validity of the 4-Disk Conjecture [22, p. 113] would imply that $\mathcal{M}_{c}$ is actually diffeomorphic to $\mathcal{D}^{4}$. However [22, p. 113], the 4-Disk Conjecture is itself equivalent [22, p. 113] to the nonexistence of exotic smooth structures on $S^{4}$. This question is currently terra incognita.

For the sake of completeness, we note that Theorem 4.1 implies Theorem 1.2 and, together with Theorem 2.2, Theorem 3.1. Our next corollary extends Brockett's necessary condition, Theorem 1.1, to the case of a compact, global attractor in $\mathbb{R}^{n}$.

Corollary 4.1. Suppose that $\mathcal{A} \subset \mathbb{R}^{n}$ is compact and that (1.2) is a smooth feedback law rendering $\mathcal{A}$ a global Liapunov stable attractor for the closed-loop system (1.3). Then, (1.4) is solvable for all $\|y\|$ sufficiently small.

EXAMPLE 4.1. [2] The classic example of a controllable system which is not asymptotically stabilizable by smooth (or continuous, Remark 1.3) feedback is Brockett's nonholonomic integrator

$$
\dot{x}_{1}=u_{1}, \quad \dot{x}_{2}=u_{2}, \quad \dot{x}_{3}=x_{2} u_{1}-x_{1} u_{2} .
$$

We note that the feedback law

$$
u_{1}(x)=x_{2}-x_{1} x_{3}, \quad u_{2}(x)=-x_{1}-x_{2} x_{3}
$$

renders the origin Liapunov stable. However, as noted in [2], for the nonholonomic 
integrator the equation (1.4) can never be solved when

$$
y=\left(\begin{array}{l}
0 \\
0 \\
\epsilon
\end{array}\right), \text { for } \epsilon \neq 0 .
$$

Therefore, the origin can be made globally Liapunov stable for the non-holonomic integrator by polynomial feedback, but (4.3) cannot be asymptotically stabilized about any compact attractor using a continuous feedback law.

REMARK 4.4. Similarly, the rigid body model of a satellite with two controls, described in Example 1.1, cannot be globally stabilized about a compact attractor $\mathcal{A}$ on $\mathbb{R}^{n}$. However, in [5] these satellite equations are stabilized about a revolute motion about an axis. In this case, there is a global attractor $\mathcal{A} \simeq S^{1}$, consisting of a submanifold of equilibria, on an ambient state-space manifold $M$ which can be shown to be diffeomorphic to $\mathbb{R}^{5} \times S^{1}$. The proof uses the $s$-Cobordism Theorem [15] of Barden, Mazur and Stallings and is similar to the proof in [4] of the corresponding result for asymptotically periodic orbits.

One consequence of Corollary 4.1 is that the compact attractor $\mathcal{A}$ must contain an equilibrium for the closed-loop system (1.3). If an equilibrium $x$ of the closed-loop vector field $F_{u}$ is isolated, then the index, $\operatorname{Ind}_{x}\left(-F_{u}\right)$, of $F_{u}$ at $x$ is defined [21] as the degree of the map

$$
\frac{-F_{u}}{\left\|F_{u}\right\|}: \partial B(x, \epsilon) \rightarrow S^{n-1}
$$

of the boundary of an $\epsilon$-ball $B(x, \epsilon)$ about $x$ to the unit sphere, for $\epsilon$ sufficiently small. If each equilibrium of (1.3) is isolated, then the Poincaré-Hopf Theorem [21], together with our main result, implies

$$
\operatorname{Ind}\left(-F_{u}\right)=\sum_{F_{u}(x)=0} \operatorname{Ind}_{x}\left(-F_{u}\right)=\chi\left(\mathcal{M}_{c}\right)=\chi\left(\mathbb{D}^{n}\right)=1 .
$$

This quantitative index formula significantly extends the existence result guaranteed by Brouwer's Fixed Point Theorem and has some interesting consequences. For example, in the equilbrium case (4.6) yields a new proof of a classical index theorem from nonlinear analysis [16].

Corollary 4.2. (Krasnosel'skii) If $G$ is a smooth vector field on $\mathbb{R}^{n}$ with $G(0)=$ 0 a locally asymptotically stable equilibrium, then

$$
\operatorname{Ind}_{0}(-G)=1 \text {. }
$$

Proof. Choose a Liapunov function $V$ for $G$ and 0 . Since 0 is the only equilibrium of $G$ in $\mathcal{M}_{c}$ for $c>0$ sufficiently small, there is only one summand in (4.6).

Definition 4.1. We shall say that an equilibrium for $-F_{u}$ is completely unstable if it is asymptotically stable for $-F_{u}$. 
REMARK 4.5. In particular, (4.7) provides the computation of the contributions to the index formula arising from the summands corresponding to an asymptotically stable equilibrium $x_{s}$, viz. $\operatorname{Ind}_{x_{s}}\left(-F_{u}\right)=1$, and to a completely unstable equilibrium $x_{c}$, viz. $\operatorname{Ind}_{x_{c}}\left(-F_{u}\right)=(-1)^{n}$.

In our next result, we will assume that any Liapunov function $V$ for a system (1.3) having a compact global attractor has been normalized by an additive constant to satisfy

$$
\left.V\right|_{\mathcal{A}}=0 \text { and } V(x)>0 \text { for } x \notin \mathcal{A},
$$

as in Definition 2.4.

Corollary 4.3. Suppose that $\mathcal{A} \subset \mathbb{R}^{n}$ is compact and that (1.2) is a smooth feedback law rendering $\mathcal{A}$ a global Liapunov stable attractor for the closed-loop system (1.3). Then, $\mathcal{A}$ contains at least one equilibrium of (1.3). If the equilibria of (1.3) are isolated, then on $\mathcal{M}_{c}$, for any $c>0$, we have

$$
\operatorname{Ind}\left(-F_{u}\right)=\sum_{F_{u}(x)=0} \operatorname{Ind}_{x}\left(-F_{u}\right)=1
$$

In particular:

1. If each equilibrium is locally asymptotically stable, then there exists a unique equilibrium for (1.3);

2. If each equilibrium is completely unstable, then there exists a unique equilibrium for (1.3), $n$ is even, and there exists a nontrivial Lagrange stable orbit in $\mathcal{A}$;

3. If each equilibrium is hyperbolic, then there are an odd number of equilibria.

Proof. Assertion (1) follows from Remark 4.5, as do the first two claims in Assertion (2). As for the final claim, denote the unique equilibrium of (1.3) by $x_{c}$. Since $x_{c}$ is completely unstable and since $\mathcal{A}$ is asymptotically stable, we must have $\mathcal{A}-\left\{x_{c}\right\} \neq \emptyset$. For any $x_{0} \in \mathcal{A}-\left\{x_{c}\right\}$, the closed-loop trajectory $\Phi\left(t, x_{0}\right)$ is Lagrange stable, by Corollary 2.1 .

For a hyperbolic equilibrium $x_{h}$, we have

$$
\operatorname{Ind}_{x_{h}}\left(F_{u}\right)=\operatorname{sign} \operatorname{det}\left(D F_{u}\left(x_{h}\right)\right)= \pm 1
$$

In particular, if each equilibrium is hyperbolic there must be an odd number of summands.

Remark 4.6. Conclusions (1) and (3) are illustrated in one dimension by the phase portrait of the pitch-fork bifurcation $\dot{x}=\mu x-x^{3}$, for $\mu<0, \mu=0$ and $\mu>0$. Conclusions (2) and (3) are illustrated in the plane by Example 2.1.

5. A Necessary Condition for Input-to-State Stability. We first recall some standard concepts concerning the comparison functions from Liapunov stability theory. 


\section{DEFINITION 5.1.}

1. A continuous function $\alpha:[0, s) \rightarrow[0, \infty)$ is said to belong to class $\mathcal{K}$, i.e. $\alpha \in \mathcal{K}$, if $\alpha$ is strictly increasing and $\alpha(0)=0$.

2. $\alpha$ is said to be of class $\mathcal{K}_{\infty}$ if $s=\infty$ and $\lim _{t \rightarrow \infty} \alpha(t)=\infty$.

3. A continuous function $\beta:[0, s) \times[r, \infty) \rightarrow[0, \infty)$ is said to belong to class $\mathcal{K} \mathcal{L}$ if $\beta(\cdot, \rho)$ is class $\mathcal{K}$ and $\beta(\sigma, \cdot)$ is monotone decreasing in $\sigma$ with $\lim _{\sigma \rightarrow \infty} \beta(\sigma, \cdot)=0$.

Suppose $\mathcal{A} \subset \mathbb{R}^{n}$ is a compact set that is invariant for (1.1) when the control $u=0$. For $x \in \mathbb{R}^{n}$, denote $\operatorname{dist}(x, \mathcal{A})$ by $\|x\|_{\mathcal{A}}$. In the theory of ISS systems it is customary to restrict attention to controls $u \in L^{\infty}\left([0, \infty), \mathbb{R}^{m}\right)$ and to denote the norm of $u$ in this space by $\|u\|$.

Definition 5.2. (Sontag) The system (1.1) is said to be input-to-state stable (ISS) with respect to $\mathcal{A}$, provided there exists a function $\beta \in \mathcal{K} \mathcal{L}$ and a function $\gamma \in \mathcal{K}$ such that

$$
\left\|x\left(t, x_{0}, u\right)\right\|_{\mathcal{A}} \leq \beta\left(\left\|x_{0}\right\|_{\mathcal{A}}, t\right)+\gamma(\|u\|) .
$$

If (1.1) is ISS with respect to a compact set $\mathcal{A}$, then by [30] there exists an ISSLiapunov function $V$ for (1.1) and $\mathcal{A}$; i.e., a smooth, proper function $V: \mathbb{R}^{n} \rightarrow \mathbb{R}$ satisfying (4.8) and

$$
\dot{V}=\langle\nabla V, f(x, u)\rangle \leq-\alpha\left(\|x\|_{\mathcal{A}}\right)+\sigma(\|u\|)
$$

for $\alpha \in \mathcal{K}_{\infty}$ and $\sigma \in \mathcal{K}$.

While the existence of an ISS-Liapunov function certainly implies that (1.1) is ISS with respect to $\mathcal{A}$, the existence of an ISS function is a substantial generalization of the converse theorems of Liapunov theory for attractors for autonomous systems, as described in Section 2. Indeed, taking $u=0$ in (5.2) shows that $V$ is a Liapunov function for (1.1) and $\mathcal{A}$, when $u=0$. In particular, any of our necessary conditions for asymptotic stability of attractors applies to such systems, but because the ISS hypotheses are more stringent, though very effective, one should expect a more sharpened version of Theorem 4.1 to hold.

TheOrem 5.1. Suppose (1.1) is ISS with respect to the compact subset $\mathcal{A} \subset \mathbb{R}^{n}$. Then, for each constant vector $d \in \mathbb{R}^{m}$, the equation

$$
f(x, d)=y
$$

is solvable for $\|y\|$ sufficiently small.

ExAmPle 5.1. In [13] it is shown that the system

$$
\dot{x}=-x+x u
$$


is not ISS-stable with respect to $\mathcal{A}=\{0\}$, despite the fact that $\{0\}$ is a globally asymptotic equilibrium of (5.4) with $u=0$. Indeed, when $d=1,(5.3)$ is solvable only for $y=0$.

EXAMPLE 5.2. In [29] it is shown that the system

$$
\dot{x}=-x+\left(x^{2}+1\right) u
$$

is not ISS-stable with respect to $\mathcal{A}=\{0\}$, despite the fact that $\{0\}$ is a globally asymptotic equilibrium of (5.4) with $u=0$. In fact, when $|d|>1 / 4$, (5.3) has no solutions.

Proof. Choose an ISS-Liapunov function $V$ for (1.1). By Theorem 4.1, $\mathcal{M}_{c}$ is homeomorphic to $\mathcal{D}^{n}$ for any $c>0$. Now fix $u=d$. Standard ISS estimates imply the existence of $\chi \in \mathcal{K}$ such that

$$
\|x\| \geq \chi(\|d\|) \Longrightarrow\langle\nabla V, f(x, d)\rangle \leq-\alpha\left(\|x\|_{\mathcal{A}}\right)
$$

Consequently, there exists $c>0$ sufficiently large so that $\mathcal{M}_{c}$ is positively invariant under the flow of the autonomous system

$$
\dot{x}=f(x, d) .
$$

Moreover, for $\|y\|$ sufficiently small, $\mathcal{M}_{c}$ is also positively invariant under the flow of the autonomous system

$$
\dot{x}=f(x, d)-y
$$

Therefore, by Theorem 4.1 and the Brouwer Fixed Point Theorem, (5.3) is solvable, for $\|y\|$ sufficiently small.

REMARK 5.1. It follows from Theorem 5.1 that there always exists an equilibrium $x_{d}$ for (1.1) forced by a constant control $u=d$, as was noted in [29] for the case $\mathcal{A}=\{0\}$. The necessary condition given by (5.3) is, however, much stronger, as shown by Example 5.1. We also note that Corollary 4.3 applies to the compact, Liapunov stable global attractor $\mathcal{A}_{d}$ that exists for the closed-loop system (5.7). In general, it is $\mathcal{A}_{d}$, rather than the union of the equilibria $x_{d}$, that should be regarded as the steady-state response of (1.1) to constant forcing $u=d$, see [14].

\section{A Necessary Condition for Practical Stabilization.}

Definition 6.1. [13] The system (1.1) is said to be practically stabilizable about the point $x^{*}$ provided that for every bounded neighborhood $x^{*} \in N \subset \mathbb{R}^{n}$ and every $\epsilon>0$ there exists a feedback control law $u=u_{\epsilon, N}(x)$ such that for every $x_{0} \in N$ there exists a $T>0$ for which the trajectory $\Phi\left(\cdot, x_{0}\right)$ of $(1.3)$ satisfying $\Phi\left(t, x_{0}\right) \in B_{\epsilon}\left(x^{*}\right)$ for $t>T$. 
Corollary 6.1. A necessary condition for the practical stabilizability of (1.1) about $x^{*}$ is that (1.4) be solvable for all $\|y\|$ sufficiently small.

Proof. Fix a bounded open subset

$$
x^{*} \in N \subset \mathbb{R}^{n}
$$

and choose $\epsilon>0$ sufficiently small such that

$$
B_{\epsilon}\left(x^{*}\right) \subset N .
$$

By assumption there exists a feedback law $u_{\epsilon, N}(x)$ such that, for the closed-loop system (1.3), $\overline{B_{\epsilon}\left(x^{*}\right)}$ attracts $N$. Set $F=F_{u_{\epsilon, N}}$ in (1.3). By [10],

$$
J=\omega(N) \subset B_{\epsilon}\left(x^{*}\right)
$$

is a compact Liapunov stable attractor for $F$ and the domain of attraction $\mathcal{D}$ of $J$ is an open subset of $\mathbb{R}^{n}$ containing $N$ (cf. [14, Lemma 4]).

On $\mathcal{D}$ we can rescale the vector field $F$ by an integrating factor so that the reparameterized trajectories of (1.3) are those of the flow $\bar{\Phi}: \mathbb{R} \times \mathcal{D} \rightarrow \mathcal{D}$ determined by a complete vector field $\bar{F}$. In particular, for any compact set $K \subset \mathcal{D}$ there exists a $T>0$ such that $K \subset \bar{\Phi}\left(-T, B_{\epsilon}\left(x^{*}\right)\right)$. As in [20] and [33], by the Brown-Stallings Theorem it follows that $\mathcal{D}$ is diffeomorphic to $\mathbb{R}^{n}$.

Since $J \subset B_{\epsilon}\left(x^{*}\right) \subset \mathcal{D}$ is a compact set that is globally asymptotically stabilizable on $\mathcal{D} \simeq \mathbb{R}^{n}$, Corollary 4.1 implies that (1.4) is solvable for all $y$ with $\|y\|$ sufficiently small.

REMARK 6.1. In particular, while the feedback law (4.4) renders the origin globally Liapunov stable for the non-holonomic integrator, (4.3) is not practically stabilizable.

\section{Necessary Conditions for Continuous Asymptotically Stabiliz-} ing Feedback Laws. Zabczyk [36] applied (4.7) to (1.3) to obtain

$$
\operatorname{Ind}_{0}\left(F_{u}\right)=(-1)^{n}
$$

whenever $u$ is a Lipschitz continuous feedback law that asymptotically stabilizes the equilibrium $x_{0}=0$. From this, he deduced Theorem 1.1 for Lipschitz continuous feedback laws by a degree theoretic result that asserts that a mapping with non-zero index (4.5) must map the interior of the ball $B(x, \epsilon)$ onto itself.

Alternatively, one can appeal to a classical result in dynamical systems, viz. the persistence of equilibria under a perturbation of an asymptotically stable equilibrium, which is also a consequence of (4.7). More precisely, for $\mu \in W$, a topological space, consider the differential equation

$$
\dot{x}=F_{\mu}(x) \quad x \in \mathbb{R}^{n},
$$


where the vector field $F_{\mu}$ is a jointly continuous family on $W \times \mathbb{R}^{n}$ of Lipschitz continuous vector fields on $\mathbb{R}^{n}$. If $F_{\mu_{0}}$ has an asymptotically stable equilibrium $x_{0}$, then for all $\mu$ sufficiently close to $\mu_{0}$,

$$
\operatorname{Ind}\left(-F_{\mu}\right)=\operatorname{Ind}\left(-F_{\mu_{0}}\right)=\operatorname{Ind}_{x_{0}}\left(-F_{\mu_{0}}\right)=1
$$

on a sublevel set $\mathcal{M}_{c}$ of a Liapunov function $V$ for $F_{\mu_{0}}$. Therefore $F_{\mu}$ has an equilibrium $x_{\mu} \in \mathcal{M}_{c}$, by degree theory [21]. In fact, $F_{\mu}$ has an asymptotically stable compact attractor $\mathcal{A}_{\mu} \subset \mathcal{M}_{c}$ which contains an equilibrium $x_{\mu}$.

Remark 7.1. For $F_{\mu}=F_{u}-\mu$ with $\mu=y$, (7.3) implies Theorem 1.1. Moreover, as a necessary condition for asymptotic stabilization, persistence of equilibrium can be shown [32] by example to be stronger than Theorem 1.1.

REMARK 7.2. If (1.3) is $C^{1}$ and has a compact attractor, then the persistence of attractors for $F_{u}-y$, for $\|y\|$ sufficiently small, will similarly imply Corollary 4.1.

In the equilibrium case, Coron ([7] and the references therein) has generalized Brockett's Theorem to the case of continuous feedback control laws by a refinement of Zabczyk's index theoretic argument [36] using topological degree theory. As it should be, in the light of Theorem 3.1 we can streamline his proof. Briefly, suppose $u$ is continuous at 0 and $\mathcal{C}^{1}$ on $\mathbb{R}^{n}-\{0\}$ and that 0 is locally asymptotically stable for (1.3). Coron begins by noting that. according to Kurzweil [17], there exists $\mathcal{C}^{\infty}$ Liapunov function $V$ for (1.3). We can apply (4.6) to the dynamical system (4.1) to obtain

$$
\operatorname{Ind}_{0}(-\nabla V)=(-1)^{n}
$$

on the smooth manifold with boundary $\mathcal{M}_{c}$, for $c>0$ sufficiently small.

It is a fundamental observation of Gauss, invoked by Coron [7], that any two inward (or two outward) pointing vector fields $X_{1}, X_{2}$ have the same index. Indeed, in modern terms, the convex combination $\lambda X_{1}+(1-\lambda) X_{2}$ is a homotopy through inward (or outward) pointing vector fields on $M_{c}$. Since both $F_{u}$ and $-\nabla V$ point inward on $M_{c}$, (7.1) holds for $F_{u}$. From (7.3) it then follows [7] that

$$
\operatorname{Ind}\left(-F_{\mu}\right)=1
$$

for $F_{\mu}(x)=F_{u}(x)-y$ and $\|y\|$ sufficiently small. In the light of our main results, we can also take $c,\|y\|$ sufficiently small so that we have a continuous map

$$
F_{\mu}: \mathcal{M}_{c} \rightarrow \mathcal{M}_{c}
$$

and a homeomorphism

$$
T: \mathcal{M}_{c} \simeq \mathbb{D}^{n},\left.\quad T\right|_{M_{c}}: M_{c} \simeq S^{n-1} \text { with } T(0)=0 .
$$


Implementing $T$ and setting $\widetilde{F_{\mu}}=F_{\mu} \circ T^{-1}$, we have the continuous map

$$
\widetilde{F_{\mu}}: \mathbb{D}^{n} \rightarrow \mathbb{D}^{n}
$$

which satisfies

$$
\operatorname{Ind}\left(-\widetilde{F_{\mu}}\right)=\operatorname{Ind}_{0}\left(-\widetilde{F_{\mu}}\right)=1 .
$$

Matters being so, (1.4) will be satisfied provided the map (7.8) takes on the value 0 .

If this were not true, then the map

$$
\frac{-\widetilde{F_{\mu}}}{\left\|\widetilde{F}_{\mu}\right\|}: S^{n-1} \rightarrow S^{n-1}
$$

would extend to the map

$$
\frac{-\widetilde{F_{\mu}}}{\left\|\widetilde{F}_{\mu}\right\|}: \mathbb{D}^{n} \rightarrow S^{n-1}
$$

Since $S^{n-1} \subset \mathbb{D}^{n}$ is contractible in $\mathbb{D}^{n}$ to a point, the degree of the composition of (7.11) with the inclusion $\iota: S^{n-1} \rightarrow \mathbb{D}^{n}$ is 0 and therefore the degree of (7.10) is 0 , contrary to (7.9) and the definition (4.5) of the index. Therefore, (1.4) has a solution.

REMARK 7.3. It is well-known that (1.4) is also a necessary condition for asymptotic stabilization by dynamic feedback compensation. Beginning with (7.1), Coron [6] has generalized Theorem 1.1 to give a new necessary condition for asymptotic stabilization by continuous dynamic feedback compensation in terms of a criterion using stable homotopy groups. This necessary condition can be shown [6] by example to be stronger than (1.4).

\section{REFERENCES}

[1] R. H. Bing, A homeomorphism between the 3-sphere and the sum of two solid horned spheres, Ann. of Math., 56(1952) 354-362.

[2] R. W. Brockett, Asymptotic stability and feedback stabilization, Differential Geometric Control Theory, R. W. Brockett, R. S. Millman, H. J. Sussmann (Eds.), Birkhäuser, Boston, 1983, pp. 181-191.

[3] M. Brown, A proof of the generalized Schoenflies Theorem, Bull. of the AMS, 66(1960), pp. 74-76.

[4] C. I. Byrnes And R. W. Brockett, Nonlinear oscillations and vector fields paired with a closed one-form, submitted to The Amer. J. of Math.

[5] C. I. Byrnes And A. Isidori, On the attitude stabilization of rigid spacecraft, Automatica, 27(1991), pp. 1122-1137.

[6] J.-M. Coron, A necessary condition for feedback stabilization, Systems Control Lett., 14(1990), pp. $227-232$.

[7] J.-M. Coron, Control and Nonlinearity, SURV 136, Amer. Math. Soc., Providence, 2007.

[8] M. H. Freedman, The topology of four-dimensional manifolds. J. Diff. Geom., 17(1982), pp. 357-453. 
[9] A. Granas and J. Dugundu, Fixed Point Theory, Springer-Verlag, New York, 2000.

[10] J. K. Hale, Asymptotic Behavior of Dissipative Systems, AMS Series: Surv Series 25, 1988.

[11] M. W. Hirsch, Differential Topology, Springer-Verlag, New York, 1976.

[12] A. Isidori, Nonlinear Control Systems, Third Ed., Springer-Verlag, New York, 1995.

[13] A. Isidori, Nonlinear Control Systems II, Springer-Verlag, New York, 1999.

[14] A. Isidori And C. I. Byrnes, Steady-state behaviors in nonlinear systems with an application to robust disturbance rejection, Annual Reviews in Control, 32(2008), pp. 1-16.

[15] M. Kervaire, Le théorème de Barden-Mazur-Stallings, Comment. Math. Helv., 40(1965), pp. $31-42$.

[16] M. A. Krasnosel'skiü and P. P. ZabreĭKo, Geometric Methods of Nonlinear Analysis, Springer-Verlag, Berlin, 1984.

[17] J. KurzweiL, On the inverse of Lyapunov's second theorem on stability of motion, Amer. Math. Transl., Ser. 2, 24(1956), pp. 19-77.

[18] J. L. Massera, Contributions to stability theory, Ann. of Math., 64(1956), pp. 182-206.

[19] B. Mazur, On embeddings of spheres, Bull. Amer. Math. Soc., 65(1961), pp. 59-65.

[20] J. W. Milnor, Differential Topology, Lectures in Modern Mathematics, (T. L. Saaty, Ed.), Vol. II. Wiley, New York, 1964.

[21] J. W. Milnor, Topology From a Differentiable Viewpoint, University Press of Virgina, Charlotsville, Virgina, 1965.

[22] J. W. Milnor, Lectures on the h-Cobordism Theorem, Princeton University Press, 1965.

[23] J. W. Morgan and G. Tian, Ricci flow and the Poincaré conjecture, Amer. Math. Soc, Clay Mathematics Monographs, Vol. 3, 2007.

[24] G. Perelman, Finite extinction time for solutions of the Ricci equation on certain three manifolds, math. DG/0303109, 2003.

[25] T. M. Price, Compact, contractible n-manifolds and their boundaries, Michigan Math. J., 18(1971), pp. 331-341.

[26] S. Smale, Generalized Poincaré Conjecture in dimensions greater than 4, Ann. of Math., 64(1956), pp. 399 - 405.

[27] S. Smale, Differentiable and combinatorial structures on manifolds, Ann. of Math., 74(1961), pp. $498-502$.

[28] S. Smale, On the structure of manifolds, Amer. J. of Math., 84(1962), pp. 387 -399.

[29] E. D. SontaG, Input-to-state stability: Basic concepts and results, Nonlinear and Optimal Control Theory, (P. Nistri, G. Stefani, eds.), Springer-Verlag, 2007, 163-220.

[30] E. D. Sontag And Y. Wang, On characterizations of input-to-state stability with respect to compact sets, Proc. of NOLCOS'95, pp. 226-231, 1995.

[31] E. H. Spanier, Algebraic Topology, McGraw-Hill, N.Y., 1966

[32] V. Sundarapandian and C. I. Byrnes, Persistence of equilibria for locally asymptotically stable systems, Int. J. of Robust and Nonlinear Control, 11(2001), pp. 87-93.

[33] F. W. WiLson, The structure of the level sets of a Lyapunov function, J. of Diff. Eqns., 3(1967), pp. 323-329.

[34] F. W. Wilson, Smoothing derivatives of functions and applications, Trans. of the Amer. Math. Soc., 139(1969), pp. $413-428$.

[35] J. A. Yorke, Periods of periodic solutions and the Lipschitz constant, Proc. of the Amer. Math. Soc., 22(1969), pp. 509-512.

[36] J. ZaвCZyк, Some comments on stabilizability, Appl. Math. Optim., 19(1989), pp. 1-19. 
